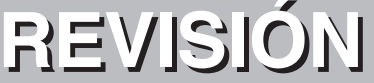

\section{Prologe: Biodegradable lubricants from vegetable oils}

\author{
By Joaquin J. Salas, M.Victoria Ruiz-Méndez y Rafael Garcés
}

In the present issue, the editorial board of this journal decided to include a series of minireviews about biolubricants. Lubrication is essential in almost every aspect that has contributed to the development of our society to the present day (industry, transportation, construction, etc). The main property of a lubricant is friction reduction or avoiding the direct contact of two surfaces in different systems. Moreover, lubricant fluids are also used for power or heat transmission in hydraulic mechanisms and heat exchanges. Biolubricants have been extensively used throughout history. They were oils and greases of both plant and animal origin (olive oil, animal fat, spermaceti oil). However, the second industrial revolution and the scarcity of sperm whales caused a complete turnover in the use of biolubricants to lubricants based on mineral oils. Mineral bases offered a higher scale of production and a higher versatility of products due the nature and the length of the carbon chains of the base which can be altered by means of petrol chemical processing. In this regard, a large majority of the bases of the lubricants used nowadays are of mineral origin. However, the extensive use of mineralbased lubricants has caused serious environmental problems due to the fact that these oils are poorly biodegradable. Thus, spills from working machines or accidents cause serious damage to ecosystems and are very contaminant. The increasing concern for environmental protection, in addition to the continuous increase in the price of crude oil have encouraged the development of newer bio-based lubricants, which are much less contaminant and involve the use of carbon chains from locally produced renewable sources. Furthermore, the latest advances in oil seed breeding and biotechnology provide new oil bases that overcome the problem of the low stability of vegetable oils. In this context, the Spanish government funded the Biovesin project, which targeted the generation of environmentally friendly lubricants from vegetable oils of the newest generation along with biodegradable additives. This project involved the participation of 14 institutions, including universities, private companies and public research institutions. This series of minireviews takes place at the end of this project and shows part of the results obtained in it. This series includes a review from Rafael Garcés and Joaquín J. Salas that aims at offering a present state of the art on the use of vegetable oils as basestocks for lubricants. In this review the advantages and disadvantages of these bases are discussed, as well as the results obtained with the new lubricants based on high oleic vegetable oils. Other aspects like additives in the oils to increase viscosity and depress pour points are also discussed. No less important is the work carried out by Terry Isbell about the use of estolides as bases for biolubricants. One of the main advantages of mineral bases over vegetable oils is their higher versatility, which involves producing bases with higher viscosities and lower pour points while maintaining high levels of stability. The production of fatty acid or triacylglycerol estolides allows for the production of biodegradable bases with improved properties and opens a promising field for the oleochemistry applied to biolubricant production. Moreover, this review examines the potential of alternative oilseeds that could be used in the future as basestocks for the production of new generation biolubricants. Finally the last review from Amaya Igartúa et al. is in the form of a technical report in which some results obtained in the Biovesin project are discussed. In this work the high level of biodegradability of added hydraulic fluids based on high oleic oils and their performance in real agricultural machinery were described. In summary, this series of minireviews embraces many aspects of the use of and production of biolubricants from oils, estolides and their potential development in the future. 